\title{
The $B_{12}$ Vitamins and Methionine in the Metabolism of Prymnesium parvum
}

\author{
By M. RAHAT* AND K. REICH \\ Department of Zoology, The Hebrew University, Jerusalem, Israel
}

(Received 31 August 1962)

\begin{abstract}
SUMMARY
Methionine or ethionine, although capable of serving as sole nitrogen source for Prymnesium parvum, cannot replace or spare the vitamin $\mathbf{B}_{12}$ nutrient requirement for the growth of this flagellate. In the presence of vitamin $B_{12}$ methionine counteracted the inhibition of growth exerted by some vitamin $\mathbf{B}_{12}$ analogues which are substituted at the benzimidazole part of the molecule. No such effect was observed against the inhibition by certain other analogues. Analogue F III ( $\alpha$-(5-hydroxybenzimidazolyl)cobamide cyanide) replaced vitamin $\mathrm{B}_{12}$ in the presence of methionine, and to a lesser degree also in the presence of other methyl donors such as methylmethionine sulphonium iodide, dimethylpropiothetin, choline chloride and betaine. The conclusion drawn is that for $\boldsymbol{P}$. parvum analogue F III is capable of replacing vitamin $B_{12}$ in all the metabolic pathways other than methyl-group synthesis. For the latter process, the benzimidazole methyl groups present in vitamin $\mathrm{B}_{12}$ and analogue F III $_{\mathrm{m}}$ ( $\alpha$-(5-methoxybenzimidazolyl)-cobamide cyanide) are indispensable.
\end{abstract}

\section{INTRODUCTION}

Vitamin $B_{12}$ participates in many biochemical reactions (see Heinrich, 1957, 1962). Davis \& Mingioli (1950) showed that vitamin $B_{12}$ is essential for the growth of a mutant of Escherichia coli and that methionine can replace the vitamin for this mutant. Helleiner, Kisliuk \& Woods (1957) and Guest, Helleiner, Cross \& Woods (1960) showed that vitamin $B_{12}$ is necessary for methionine synthesis by cell-free extracts of $E$. coli. A dependence on vitamin $\mathbf{B}_{12}$ is also known for Ochromonas malhamensis, an allied flagellate of Prymnesium parvum, the growth of which is enhanced by methionine in the presence of vitamin $\mathbf{B}_{12}$ (Hutner, Provasoli \& Filfus, 1953; Johnson, Holdsworth, Porter \& Kon, 1957; Ford, 1958). The growth of $O$. malhamensis is inhibited by ethionine, but this inhibition can be annulled by methionine. High concentrations of methionine, however, are also inhibitory (Johnson et al. 1957).

Vitamin $\mathrm{B}_{12}$ is an essential nutrient for Prymnesium parvum (Droop, 1954; Rotberg, 1958), and may be replaced by some of its analogues, while other analogues inhibit the growth of $\boldsymbol{P}$. parvum even in the presence of vitamin $\mathbf{B}_{12}$ (Rahat \& Reich, 1962). To date, no compound has been found which can replace vitamin $B_{12}$ for $\boldsymbol{P}$. parvum, except the few related compounds just mentioned. Though some amino acids may serve as sole nitrogen source for $\boldsymbol{P}$. parvum, vitamin $\mathbf{B}_{12}$ is still an

* Present address: Department of Biochemistry, Medical School, Vanderbilt University, Nashville, Tennessee. 
essential nutrient (Rahat \& Reich, 1963). It is the purpose of the present paper to discuss the function of vitamin $\mathbf{B}_{12}$ and some of its analogues in the metabolism of $\boldsymbol{P}$. parvum, and the possible role of this vitamin in the synthesis of methionine and free methyl groups by this flagellate.

\section{METHODS}

The Reich strain of Prymnesium parvum was used in the present work. Data on medium composition and methods of culture are given elsewhere (Rahat \& Reich, 1963). The amount of growth of $P$. parvum was determined at the end of 12 days by measuring the optical density of a culture with a Junior Coleman Spectrophotometer at $480 \mathrm{~m} \mu$. A list of vitamin $B_{12}$ analogues used in the present work is given in Table 1.

Table 1. Compounds of the vitamin $\mathrm{B}_{12}$ group used in the present work

In this paper vitamin $B_{12}$ means $\alpha$-(5, 6-dimethylbenzimidazolyl) cobamide cyanide. Other analogues are presented by name and code.

Abbreviation used

1. $B_{12}$

2. FIII

3. FIII $_{m}$

4. $\mathrm{PVB}_{12}$

5. FA

6. 2MMA Cob

7. EAM

8. MA

9. An
The analogues

Vitamin $\mathbf{B}_{12}$ commercial, Nutritional Biochemical Co., U.S.A.; analogues 2-6 received from K. Bernhauer, Technische Hochschule, Stuttgart, Germany; analogues 7-9 received from E. Lester Smith, Glaxo Laboratories, England.

\section{RESULTS}

Experiment showed that methionine and ethionine could be used by Prymnesium parvum as sole nitrogen sources. No growth inhibition occurred with either of these amino acids even at high concentrations (Fig. 1). In a second series of experiments, almost identical growth curves were obtained when the above-mentioned amino acids were added at concentrations of $0.25 \mathrm{mg} . / \mathrm{ml}$. (Fig. 2) and $2.0 \mathrm{mg} . / \mathrm{ml}$. Neither methionine nor ethionine show such sparing of vitamin $\mathbf{B}_{12}$ for $\boldsymbol{P}$. parvum as is known for Escherichia coli (Davis \& Mingioli, 1950) and Ochromonas malhamensis (Johnson et al. 1957). Even though no sparing of vitamin $\mathrm{B}_{12}$ was detected, nevertheless methionine, when added to media containing vitamin $\mathbf{B}_{\mathbf{1 2}}$ and growthinhibitory analogues, was found to counteract the inhibition caused by some of these analogues (Fig. 4). This annulment of inhibition by methionine was clearly evident when the compounds FIII, PVB $_{12}$, 2MMA Cob and FA were the inhibitors; these are substituted in the nucleotide part of the molecule. However, with inhibitory analogues which were substituted in the propionamide of the corrinoid part of the molecule (e.g. compounds MA, EAM, An), only a slight annulment of inhibition was observed, which may have been due merely to the addition of another organic metabolite. 
Table 2 shows the growth of Prymnesium parvum in media devoid of vitamin $\mathbf{B}_{12}$, in the presence of the inhibitory analogues and with or without methionine. It can be seen at once that of all the analogues tested only F III replaced vitamin $B_{12}$ in the presence of methionine. As pointed out earlier only analogues substituted in the

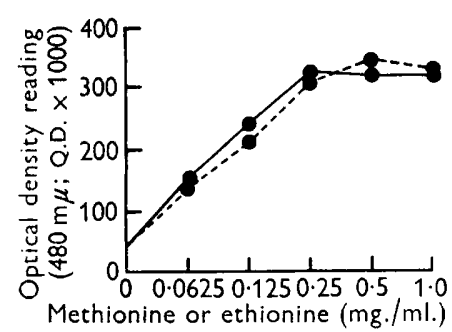

Fig. 1

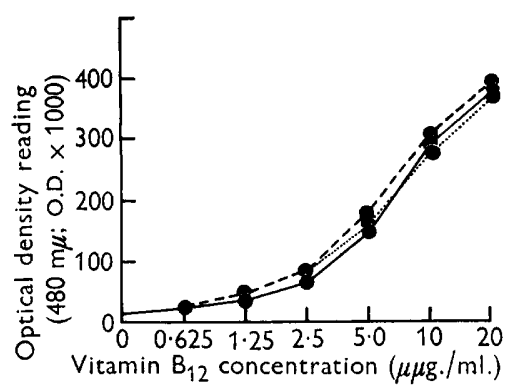

Fig. 2

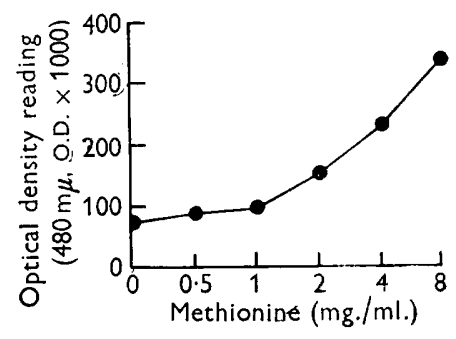

Fig. 3

Fig. 1. Growth of Prymnesium parvum in inorganic medium with methionine or ethionine as sole nitrogen source. In these experiments Casamino acids were omitted from the basal medium (see Rahat \& Reich, 1962). —-, Methionine; - - -, ethionine.

Fig. 2. Growth of Prymnesium parvum at various suboptimal concentrations of vitamin $B_{12}$ in the presence of methionine or ethionine $(0.25 \mathrm{mg} . / \mathrm{ml}) . \quad-$.- , Vitamin $B_{12}$; - , vitamin $\mathrm{B}_{12}+$ methionine; ....., vitamin $\mathrm{B}_{12}+$ ethionine.

Fig. 3. Growth of Prymnesium parvum at various concentrations of methionine, in the presence of compound FIII $\left(10 \mathrm{~m} \mu \mathrm{g} . / \mathrm{ml}\right.$.) with vitamin $B_{12}$ omitted.

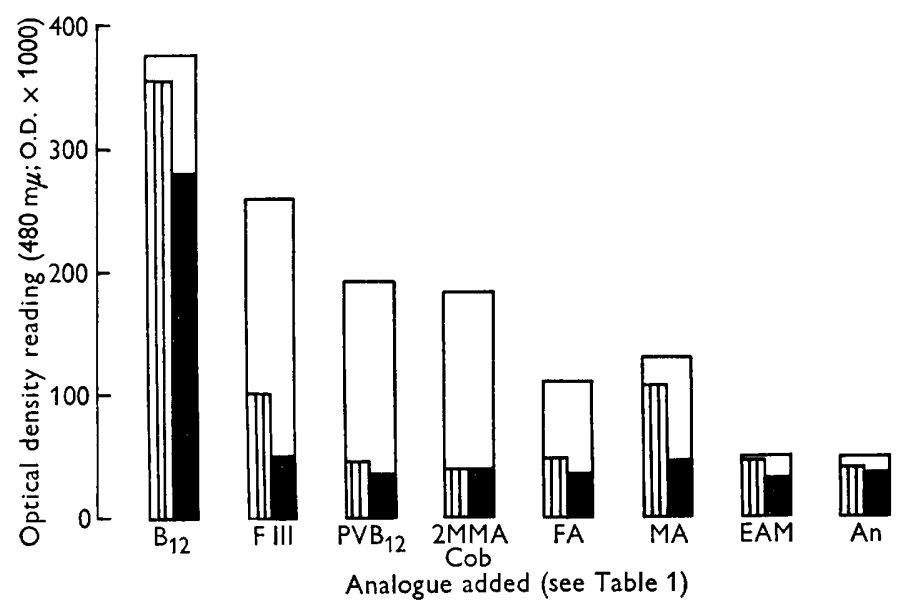

Fig. 4. Effect of methionine or ethionine $(4 \mathrm{mg} . / \mathrm{ml}$.) on the growth of Prymnesium paroum in the presence of vitamin $\mathbf{B}_{12}$ and growth inhibitory analogues. Vitamin $\mathbf{B}_{12}$ was added at $100 \mu \mu \mathrm{g} . / \mathrm{ml}$. and the respective analogues at $100 \mathrm{~m} \mu \mathrm{g} . / \mathrm{ml}$. Broad open column $=$ with methionine; right half (solid black) $=$ with ethionine; left half (vertical rules) $=$ no addition.

nucleotide part of the molecule had their growth-inhibitory effect annulled by methionine. Of these analogues, only F III has the benzimidazole group unchanged, whereas in compounds FA, $\mathbf{P V B}_{12}$ and 2MMA Cob (see Table 1), this group is replaced by adenine or its derivative. 
In the absence of vitamin $B_{12}$ but with $10 \mathrm{~m} \mu \mathrm{g}$. F III $/ \mathrm{ml}$. $+8 \mathrm{mg}$. methionine $/ \mathrm{ml}$, growth of Prymnesium parvum was equal to that obtained with vitamin $\mathbf{B}_{12}$ alone (Fig. 3). Higher concentrations of compound FIII gave no additional growth. Noteworthy, however, is the fact that, whereas growth of Ochromonas malhamensis is inhibited by $0.8 \mathrm{mg}$. methionine $/ \mathrm{ml}$., for $\boldsymbol{P}$. parvum maximal growth was attained in the presence of $8 \mathrm{mg}$. methionine/ml. An attempt was made to replace methionine, in the presence of compound FIII, by other potential methyl donors with homocysteine added as a methyl acceptor. As can be seen from Table 3, these methyl donors exerted an effect qualitatively similar to that of methionine.

Table 2. Growth of Prymnesium parvum in the absence of vitamin $\boldsymbol{B}_{12}$ but \pm methionine and various analogues

\begin{tabular}{lcc}
\multicolumn{1}{c}{$\begin{array}{c}\text { Optical density reading } \\
\text { Analogues }\end{array}$} & $\begin{array}{c}\text { With } \\
\text { methionine }\end{array}$ & $\begin{array}{c}\text { Without } \\
\text { methionine }\end{array}$ \\
(at 2 m $\mu$ g./ml.) & $(4 . \mathrm{mg} . / \mathrm{ml}$.) & \\
FA & 13 & 8 \\
FIII & 268 & 50 \\
2MMA Cob & 18 & 13 \\
PVB & 13 & 8 \\
EAM & 13 & 18 \\
MA & 22 & 22 \\
An & 31 & 18
\end{tabular}

Table 3. Growth of Prymnesium parvum in the presence of compound FIII ( $\alpha$ [5-hydroxybenzimidazolyl]-cobamide cyanide; $10 \mathrm{~m} \mu \mathrm{g} . / \mathrm{ml}$.) and potential methyl-donors with homocysteine as methyl acceptor

\begin{tabular}{lr}
\multicolumn{2}{c}{ Optical densit } \\
Methyl-donor added & (o.D. $\times 1$ \\
MMT & 65 \\
MMT + HCSTE & 114 \\
Thet & 60 \\
Thet + HCSTE & 108 \\
Ch & 91 \\
Ch + HCSTE & 114 \\
Bt & 75 \\
Bt+ HCSTE & 119 \\
HCSTE & 65 \\
No addition & 46
\end{tabular}

MMT = Methylmethionine sulphonium iodide $(100 \mu \mathrm{g} . / \mathrm{ml}$.$) ; Thet =$ Dimethyl propiothetin HCl $(100 \mu \mathrm{g} . / \mathrm{ml}.) ; \mathrm{Ch}=$ Choline chloride $(1.0 \mathrm{mg} . / \mathrm{ml}.) ; \mathrm{Bt}=$ Betaine $(1.0 \mathrm{mg} . / \mathrm{ml}$. $) ;$ HCSTE = Homocysteine $(100 \mu \mathrm{g} . / \mathrm{ml}$.).

It is known that vitamin $B_{12}$ participates in nucleic acid metabolism (Dinning \& Young, 1960; Manson, 1960; Downing \& Schweigert, $1956 a, b)$. On the assumption that compound FIII in presence of methionine might be replaced by a nucleotide, various nucleotides were added to basal media free from any members of the vitamin $B_{12}$ group. The nucleotides tested, at $0 \cdot 1 \mathrm{mg} . / \mathrm{ml}$., were : adenine, adenosine, deoxyadenosine, adenylic acid, cytidine, cytosine, cytidylic acid, guanosine, thymine, thymidine, uridine, RNA, DNA. No growth occurred in any culture tested. 


\section{DISCUSSION}

Methionine and ethionine can both be used by Prymnesium parvum as sole nitrogen source. Even at high concentrations, neither of these two amino acids exerts on $P$. parvum the growth inhibitory effect they can exert on Ochromonas malhamensis (Johnson et al. 1957). This may possibly be attributable to a low uptake by $\boldsymbol{P}$. parvum of methionine and ethionine into sites where they are required in unchanged form, whereas at sites where they function as catabolic substrates these amino acids may be utilized even at high concentrations. Since with $\boldsymbol{P}$. parvum there is no antagonistic interaction between methionine and ethionine, it must be assumed that the metabolic system of this organism is either so specific as to prevent ethionine from interfering with the action of methionine, or is such as to utilize both amino acids equally well, enabling ethionine to be incorporated into cell protein, as is the case with Tetrahymena (Gross \& Tarver, 1955).

The addition of methionine, but not of ethionine, to media containing vitamin $B_{12}$ and one of the growth-inhibitory benzimidazole-substituted analogues annulled the inhibitory effect of the latter. There was no such annulment in cultures in which the inhibitory analogues were ones substituted in the corrinoid part of the molecule. This shows that the various inhibitory analogues exert their inhibitory effects at different sites. The benzimidazole-substituted analogues apparently inhibit methionine or methyl-group synthesis, and therefore their inhibition is annulled when methionine is added. The propionamide-substituted analogues seem to inhibit some other biosynthetic pathway. To obtain the above-mentioned annulment, a relatively high concentration of methionine was required. This also might be explained by assuming a low uptake of methionine as such, at the sites where it is needed unchanged. Perhaps it is at these sites that methionine is synthesized when vitamin $B_{12}$ is not subject to competitive inhibition of an inhibitory analogue. Methionine, while obviously synthesized only in the presence of vitamin $\mathbf{B}_{12}$, shows no sparing effect for this vitamin. It seems likely therefore that only a small part of the vitamin $B_{12}$ is utilized in methyl metabolism, the rest participating in other metabolic processes.

As mentioned before, the growth of Prymnesium parvum is inhibited by benzimidazole-substituted analogues (e.g. compounds FA, PVB ${ }_{12}$ and 2MMA Cob, Table 1 ) even in the presence of vitamin $B_{12}$; however, the addition of methionine enables growth. This suggests that, of the functions of vitamin $\mathbf{B}_{12}$ in the organism, only that of methionine synthesis is inhibited by these analogues. Hence the addition of methionine enables growth of $\boldsymbol{P}$. parvum even in the presence of such inhibitory analogues. For Ochromonas malhamensis the inhibition was shown to be exerted on the uptake of the vitamin (Ford, 1958).

Such a mechanism of action of $\mathrm{B}_{12}$ antagonists cannot be excluded in $P$. parvum. A final conclusion whether Ochromonas and Prymnesium are different in this respect will have to await further experiments on both organisms.

Compound F III $_{m}$ (-5-methoxy) replaces vitamin $\mathbf{B}_{12}$ for Prymnesium parvum while compound FIII (-5-hydroxy) inhibits growth, even in the presence of vitamin $B_{12}$ (Rahat \& Reich, 1963). However, compound F III in the presence of methionine replaces vitamin $\mathbf{B}_{12}$ for $\boldsymbol{P}$. parvum. No precursors of methionine or allied amino acids tested had such effect with compound FIII. This emphasizes the importance 
of the methyl groups in methionine, compound $\mathrm{FIII}_{\mathrm{m}}$ and vitamin $\mathrm{B}_{12}$. There is, however, the possibility that compound F III in the presence of labile methyl groups of methionine may be converted to $\mathrm{FIII}_{\mathrm{m}}$ or even to vitamin $\mathrm{B}_{12}$ proper. If this be so, then the reverse transfer of methyl groups should be possible, i.e. transformation of a methionine precursor into methionine as a result of transmethylation of the vitamin $\mathbf{B}_{12}$ benzimidazole methyl groups. In $P$. parvum cultures containing compound FIII instead of vitamin $B_{12}$, the addition of various methyl donors (methylmethionine sulphonium iodide, dimethylpropiothetin, choline, betaine) in the presence or absence of homocysteine as methyl acceptor gave enhancement of growth, qualitatively like that attained with vitamin $B_{12}$. This again emphasizes the importance of free methyl groups for the growth of $\boldsymbol{P}$. parvum in the absence of vitamin $B_{12}$, which obviously participates in the synthesis of these groups. Compound FIII has a benzimidazole moiety in its molecule, and can replace vitamin $\mathbf{B}_{12}$ in the presence of methionine. In the other nucleotide-substituted analogues tested the benzimidazole moiety was replaced by a purine group. It would seem, therefore, that compound FIII can assume all the functions of vitamin $B_{12}$ except that of methionine or methyl group synthesis, for which the methyl groups of the benzimidazole moiety are indispensable. The latter syntheses are actually inhibited by the presence of hydroxybenzimidazole moiety in the molecule of FIII. Thus, the provision of methionine can relieve vitamin $B_{12}$ from one of its functions, methionine synthesis, and compound FIII can assume the remaining functions of the vitamin. Attempts to find a compound that can replace F III in the presence of methionine have thus far been unsuccessful.

The authors are indebted to Professor K. Bernhauer, Technische Hochschule, Stuttgart, Germany, and to Dr E. Lester Smith, Glaxo Laboratories, England, for the generous donation of the analogues of vitamin $B_{12}$ used in the present work.

\section{REFERENCES}

Davis, B. D. \& Mingrou, E. S. (1950). Mutants of Escherichia coli requiring methionine or vitamin $\mathrm{B}_{12}$. J. Bact. $60,17$.

Dinning, J. S. \& Young, R. S. (1960). Some effects of folic acid and vitamin $B_{12}$ on nucleic acid metabolism in Lactobacillus leichmannii. J. biol. Chem. 235, 3008.

Downing, M. \& Schweigert, B. S. (1956a). Role of vitamin $B_{12}$ in nucleic acid metabolism. III. Preparation of $\mathrm{C}^{14}$ uniformly labeled pyrimidine deoxynucleosides. J. biol. Chem. 220, 513.

Downing, M. \& Schweigert, B. S. $(1956 b)$. Role of vitamin $B_{12}$ in nucleic acid metabolism. IV. Metabolism of $\mathrm{C}^{14}$-labeled thymidine by Lactobacillus leichmannii. J. biol. Chem. 220, 521.

Droop, M. R. (1954). Cobalamin requirement in Chrysophyceae. Nature, Lond. 174, 520.

Fond, J. E. (1958). $\mathrm{B}_{12}$ vitamins and growth of the flagellate Ochromonas malhamensis. J. gen. Microbiol. 19, 161.

Gross, D. \& Tarver, H. (1955). Studies on ethionine. IV. The incorporation of ethionine into the proteins of Tetrahymena. J. biol. Chem. 217, 169.

Guest, J. R., Helueiner, C. W., Cross, M. J. \& Woods, D. D. (1960). Cobalamin and the synthesis of methionine by ultrasonic extracts of Escherichia coli. Biochem. J. 76, 396.

Heinrich, H. C. (editor) (1957). Vitamin $B_{12}$ und Intrinsic Factor. 1. Europäisches Symposion, Hamburg 1956. Stuttgart: Ferdinand Enke Verlag. 
HeINRICH, H. C. (editor) (1962). Vitamin $B_{12}$ und Intrinsic Factor. 2. Europäisches Symposion, Hamburg 1961. Stuttgart: Ferdinand Enke Verlag.

Helleiner, C. W., Kisliuk, R. L. \& Woods, D. D. (1957). Cobalamin and the synthesis of methionine by cell free extracts of Escherichia coli. J. gen. Microbiol. 18, 23.

Hutner, S. H., Provasoli, L. \& Filfus, J. (1953). Nutrition of some phagotrophic freshwater Chrysomonads. Ann. N.Y. Acad. Sci. 56, 852.

Johnson, B. C., Holdsworth, E. S., Porter, J. W. G. \& Kon, S. K. (1957). Vitamin $\mathrm{B}_{12}$ and methyl-group synthesis. Brit. J. Nutr. 2, 313.

Manson, L. A. (1960). Vitamin $B_{12}$ and deoxyribose synthesis in Lactobacillus leichmannii. J. biol. Chem. 235, 2955.

Rahat, M. \& Reich, K. (1963). The $\mathrm{B}_{12}$ vitamins and growth of the flagellate Prymnesium parvum (Chrysomonadina). J. gen. Microbiol. 31, 195.

Rotberg, M. (1958). The thiamine requirements of Prymesium parvum (Chrysomonadina). Bull. Research Council Israel. 7в, 199. 